\title{
Lung uptake during 99mTc-hydroxymethylene diphosphonate scintigraphy in patient with TTR cardiac amyloidosis: An underestimated phenomenon is
}

\author{
Francesco Cappelli a,b,*, Chiara Gallini ${ }^{c}$, Egidio Natalino Costanzo ${ }^{c}$, Francesca Tutino ${ }^{c}$, Alfonso Ciaccio ${ }^{c}$, \\ Luca Vaggelli $^{\mathrm{c}}$, Simone Bartolini ${ }^{\mathrm{b}}$, Sofia Morini ${ }^{\mathrm{b}}$, Raffaele Martone ${ }^{\mathrm{b}}$, Paola Angelotti ${ }^{\mathrm{a}}$, Sabrina Frusconi ${ }^{\mathrm{a}}$, \\ Carlo Di Mario $^{\mathrm{b}}$, Federico Perfetto ${ }^{\mathrm{a}}$ \\ a Tuscan Regional Amyloid Center, Careggi University Hospital, Largo Brambilla 3, 50134 Florence, Italy \\ b Interventional Structural Cardiology Division, Careggi University Hospital, Largo Brambilla 3, 50134 Florence, Italy \\ c Nuclear Medicine Division, Careggi University Hospital, Largo Brambilla 3, 50134 Florence, Italy
}

\section{A R T I C L E I N F O}

\section{Article history:}

Received 28 May 2017

Received in revised form 25 September 2017

Accepted 6 October 2017

\section{Keywords:}

99mTc-HMDP

TTR amyloidosis

Lung uptake

\begin{abstract}
A B S T R A C T
Background: Full body scintigraphy using bone tracers plays an important role in defining the type of amyloidosis and in diagnosing the heart involvement (cardiac amyloidosis, CA). No study has been conducted to explore lung retention (LR) in CA and its correlation to heart retention (HR).We evaluated LR in patients undergoing 99mTcHMDP scintigraphy during evaluation for suspected CA.

Methods and results: We enrolled 93 suspected CA patients. Patients underwent a complete diagnostic work up. After diagnostic process 82 patients resulted affected by certain CA (20 AL and 62 TTR), while 11 subjects showed left ventricular hypertrophy (LVH) not caused by CA. 99mTc-HMDP cardiac uptake was evaluated using the Perugini visual score while the modified Janssen score was used for LR estimation (grade 0 no uptake, grade 1 less than ribs, grade 2 more than ribs).

Results: 99mTc-HMDP LR was observed in 1/20 AL patient (5\%), while 36/62 (58\%) TTR patients showed LR with 29 grade 1 (47\%) and 7 grade 2 (11\%). No LR was observed in patients with LVH and no CA. LR was not evident in patients without HR, present in 1/3 (33\%) of the patients with Perugini 1 HR and 11/24 (46\%) and 26/36 (72\%) of the patients showing respectively a Perugini 2 and a Perugini 3.

Conclusion: 99mTc-HMDP scintigraphy shows LR in about 60\% of TTR subjects, related to the grade of $\mathrm{HR}$. In AL amyloidosis LR is less frequent than in TTR amyloidosis suggesting an aetiological tropism that seems comparable to the already known TTR related cardiac tropism.
\end{abstract}

(C) 2017 Elsevier B.V. All rights reserved.

\section{Introduction}

Amyloidoses are rare diseases characterized by extracellular deposition of protein-derived fibrils which show apple-green birefringence when stained with Congo red and viewed under polarized light in various tissues and organs, including the heart [1].

Full body scintigraphy using bone tracers plays an important role in defining the type of amyloidosis and in diagnosing the heart involvement (cardiac amyloidosis, CA) [2-10].

\footnotetext{
is All authors take responsibility for all aspects of the reliability and freedom from bias of the data presented and their discussed interpretation.

* Corresponding author at: Intensive Cardiac Care Unit, Department of Heart Lung and Vessels, Azienda Ospedaliero-Universitaria Careggi [AOUC], Largo Brambilla 3, 50134 Florence, Italy.

E-mail address: cappellifrancesco@inwind.it (F. Cappelli).
}

Pathologically, involvement in the amyloid deposition of the respiratory tract is common [11-14] and may involve the lungs in 4 distinct forms: lymphatic, diffuse alveolar septal, nodular parenchymal, and tracheobronchial, with the former two patterns commonly present in patients with systemic involvement and the latter two occurring more commonly in localized forms. Systemic pulmonary amyloidosis is frequently asymptomatic and dyspnoea, which is the leading symptom, is mainly attributed to simultaneous cardiac involvement and heart failure.

Few reports have mentioned in the past bone tracers lung retention both in patients with AL and TTR amyloidosis [15-18]. Nevertheless no comprehensive study has been conducted to assess lung uptake in systemic amyloidosis and its correlation with cardiac uptake. Moreover to the best of our knowledge no study compared incidence and severity of bone tracer scintigraphy lung retention in different aetiologies of systemic amyloidosis.

Therefore the aim of our research has been to evaluate the presence and grade of lung retention and its correlation to heart retention in a 
cohort of patient undergoing 99mTc-HMDP scintigraphy in a multidisciplinary evaluation for suspected cardiac amyloidosis.

\section{Material and method}

From the Tuscan regional amyloidosis centre database we retrospectively evaluated all patients that underwent to 99mTc-HMDP total body scintigraphy as part of the usual diagnostic workup to exclude or confirm a suspected cardiac amyloidosis. All patients underwent physical examination, ECG, transthoracic echocardiography and blood tests including NT-proBNP, serum and urinary immunifixation and serum free light chain. In case of inconclusive results patients underwent cardiac MRI.

All patients gave written informed consent for their clinical records to be used for research purposes, in accordance with Institutional Review Board guidelines.

\subsection{Diagnosis of amyloidosis and cardiac involvement definition}

Diagnosis of light-chain (AL) amyloidosis was made by biopsy of abdominal fat pad o biopsy of an involved organ, which demonstrated the typical Congo Red birefringence when viewed under polarized light. All positive biopsies demonstrated typical Congo Red birefringence under polarized light and staining by anti-k or anti- $\lambda$ light-chain antibodies, combined with elevated serum or urine levels of the corresponding monoclonal light chain.

Diagnosis of TTR amyloidosis was based on tissue biopsy with anti-TTR antibody staining (irrespectively to bone scintigraphy cardiac uptake) or, in the absence of positive tissue biopsy according to Gillmore et al. [19], by a Perugini grade 2 or 3 cardiac uptake on a bone tracer radionuclide, and absence of a detectable monoclonal protein, in a patient with echocardiogram and/or MRI suggestive of amyloidosis. Diagnosis of TTRm or TTRwt was made according to the presence or absence of mutation at genotyping.

Diagnosis of cardiac amyloidosis was made according to the International Society of Amyloidosis criteria [20].

Patients were considered to have left ventricular hypertrophy (LVH) not related to amyloidosis infiltration if they had a negative cardiac biopsy or at least 2 negative non-cardiac biopsies with no suggestive signs of cardiac amyloidosis at non-invasive imaging evaluation (99mTc-HMDP total body scintigraphy, TTE and MRI) and absence of amyloidogenic mutation at genotyping. In those patients further research to assess a cause of LVH was performed.

\subsection{Standard and tissue Doppler imaging echocardiography}

Patients were referred to our laboratory for echocardiographic study. Echocardiography was performed using a Vivid 9 System (Vingmed, General Electric, Horten, Norway) equipped with a $3 \mathrm{~S}$ probe. According to the standards of the American Society of Echocardiography [21], the following parameters were assessed: end-diastolic thickness of interventricular septum (IVS) and LV posterior wall (PW), LV end-diastolic and endsystolic volumes (LVEDV and LVESV, respectively), ejection fraction (estimated with the biplane Simpson method), mitral peak flow velocity in early and late diastole (E and A, respectively), E-wave deceleration time, E/A ratio, systolic displacement of the lateral portion of the tricuspid annular plane systolic excursion (TAPSE). We also evaluated pulsed tissue Doppler imaging-derived early diastolic peak velocity at mitral annulus ( $\left.E^{\prime}\right)$, and $E / E^{\prime}$ ratio as an index of LV filling pressure. Pulmonary artery systolic pressure (PASP) was approximated by adding to trans-tricuspid pressure gradient an estimate of right atrial pressure assessed by inferior vena cava dimension and respiratory variation.

\subsection{9mTc-HMDP scintigraphy}

All patients had 99mTc-HMDP whole body scintigraphy (HMDP-TB). 99mTc-HMDP was prepared from the commercial kit (OSTEOCIS ${ }^{\circledR}$ ) following the prescriptions of the manufacturer.

All the preparations were tested for total hydrolysed and free $99 \mathrm{mTc}(<5 \%)$ and for radiochemical purity $(>95 \%)$

Each patient received intravenous 700-740 MBq of 99mTc-HMDP and a whole body scan (anterior and posterior projections) was performed 150 min later in a $256 * 1024$ matrix. Images were acquired in different gamma cameras using low energy, high resolution collimators and an appropriate scan speed to reach over 2,000,000 count. [2].

Each scintigraphy was evaluated with Perugini visual score as previously published

For lung uptake analysis a three degree modified and simplified Janssen [22] visual score system was used: level 0 no significant lung uptake; level 1 light diffuse lung uptake less than ribs; level 2 high and/or inhomogeneous lung uptake (Fig. 1). This grading system resulted adequate to classify all patients of our series.

\subsection{Chest $X$-ray radiological findings}

In 49 patients, a recent chest X-ray (within 6 months from scintigraphy) was available and analyzed. Radiological findings were classified according to a three level score: level 0 no significant interstitial changes; level 1 presence of modest/moderate interstitial thickening or striations, no nodules; level 2 presence of significantly greater interstitial thickening and/or striations and/or nodules.

\section{Statistical analysis}

Continuous variables are expressed as median values and standard deviation, and categorical variables as frequencies and percentages. Multiple comparisons for continuous variables were performed with ANOVA followed by Scheffè test for post hoc analyses. Categorical comparisons were performed by means of the Pearson $\chi^{2}$ test.

All statistical analyses and comparisons were performed with SPSS 20 Version IBM Package and we considered statistical significance a p value $<0.05$.

\section{Results}

Our study population consisted in 93 patients with suspected cardiac amyloidosis. According to the above-mentioned criteria 82 patients resulted affected by certain cardiac amyloidosis while after complete diagnostic workup 11 patients showed LVH not amenable to amyloid infiltration. In this subgroup 9 patients showed a hypertensive cardiomyopathy while in 2 patients a genetic confirmed diagnosis of hypertrophic cardiomyopathy (HCM) was obtained.

Among patient with cardiac amyloidosis 20 patients had AL, 20 had TTRm (6 patients with Val122Ile and 14 with Ile68Leu mutation), and 42 patients were affected by TTRwt. In AL patients, biopsy site was abdominal fat in 19 patients (95\%), myocardium in 1 (5\%).

In TTRm a positive biopsy was present in $16 / 20$ patients and the positive biopsy site was abdominal fat in 11 patients (68\%), myocardium in 5 (32\%). In TTRwt a positive biopsy was present in 10/42 patients and the positive biopsy site was abdominal fat in 5 patients, myocardium in 5 .

Demographic and clinical characteristics of the three groups are summarized in Table 1.

As expected significant difference in age was observed in the studied population, with younger patients in the AL group. No significant difference was observed between groups in heart failure symptoms evaluated with New York Heart failure Association (NYHA) scale. Similar echocardiographic variables were observed among the various groups except for a significant increase in LV PW thickness in TTR patients compared to LVH subjects.

\subsection{9mTc-HMDP scintigraphy}

As shown in Table 2 in AL cardiac amyloidosis, only 1 patient demonstrated a mild heart retention at 99mTc-HMDP scintigraphy, Perugini score grade 1 , while 19 patients showed no bone tracer cardiac uptake. On the other hand all patients with TTR cardiac amyloidosis showed 99mTc-HMDP heart uptake with 2 subjects showing a grade 1 Perugini visual score, 24 subjects a grade 2 and 36 subjects a grade 3 . No heart retention was evidenced in patients with LVH but no CA.

One patient with AL amyloidosis (5\% of AL group) demonstrated mild lung retention, grade 1 visual score, while 36 (58\%) TTR patients showed pulmonary uptake with 29 grade 1 and 7 grade 2 lung retention. No lung retention was evidenced in LVH subjects.

On a subgroup analysis lung uptake was not evident in patients without cardiac uptake, present in $1 / 3(33 \%)$ of the patients with Perugini 1 cardiac uptake and 11/24 (46\%) and 26/36 (72\%) of the patients showing respectively a Perugini 2 and Perugini 3 cardiac uptake $(\mathrm{p}<0.001$, Fig. 2$)$

Pulmonary uptake was uniform in all but one patient, without visually evident gradient between apical or basal portion of the lung. Only one patient showed an inhomogeneous lung bone tracer retention with patchy areas of hyperactivity.

Despite available only in a proportion of the studied population, non-significant difference in radiological pulmonary score was evidenced between groups. 


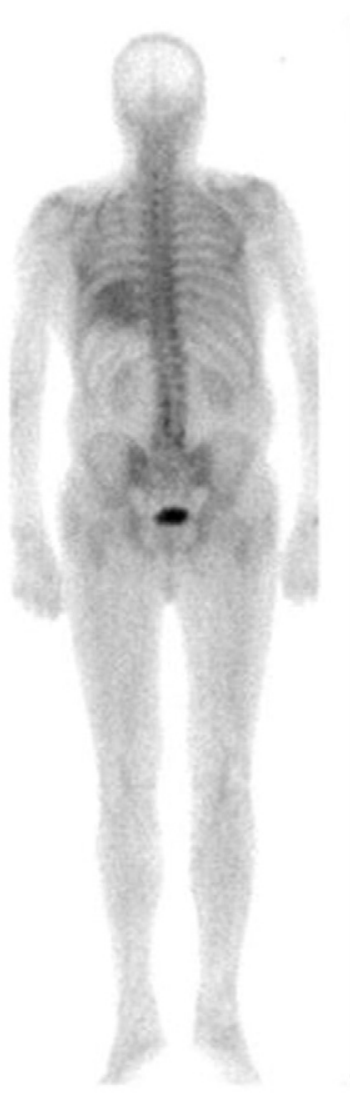

Heart uptake:

grade 1

level 0

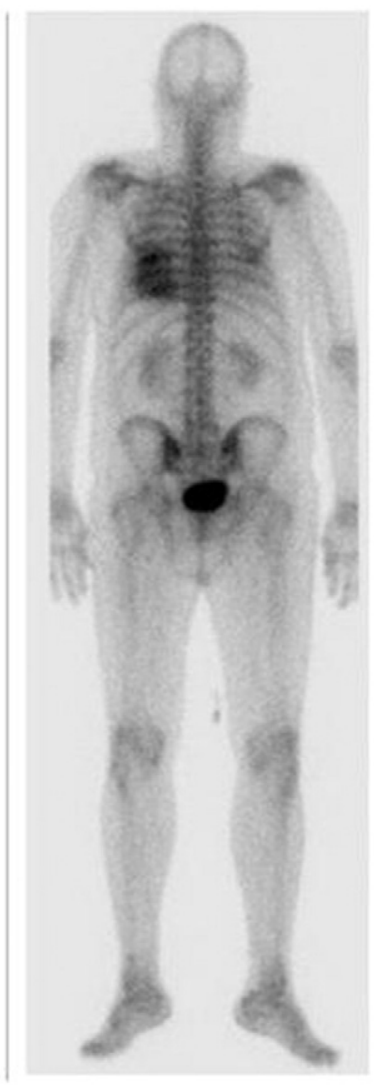

grade 2

level 1

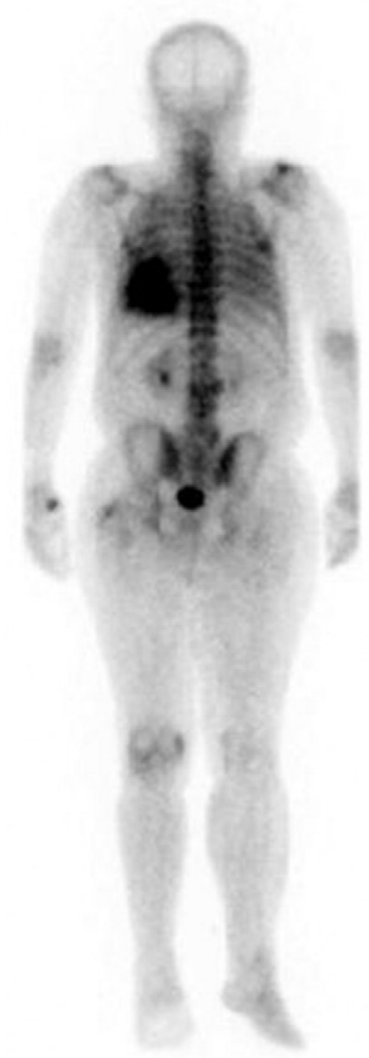

grade 2

level 2

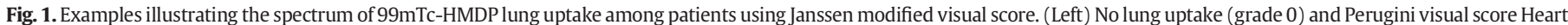

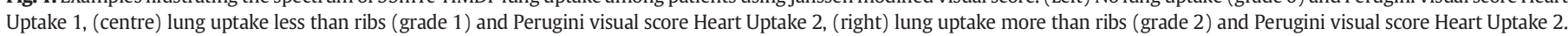

\section{Discussion}

In recent years radioactive bone tracers to diagnose cardiac amyloidosis and amyloid typing have been extensively studied [2-4,19].

On the other hand the presence of bone tracer lung retention in patients with AL and TTR amyloidosis has been far less investigated.

Table 1

Demographic and clinical characteristics.

\begin{tabular}{lllll}
\hline & AL $(\mathrm{n}=20)$ & TTR $(\mathrm{N}=62)$ & LVH $(\mathrm{N}=11)$ & $\mathrm{p}=$ \\
\hline Age & $73.1 \pm 11.9$ & $79 \pm 6.5$ & $78.8 \pm 8.7$ & 0.02 \\
Gender M/F & $14 / 6$ & $52 / 10$ & $10 / 1$ & 0.26 \\
NYHA $\geq 3$ & $12 / 20(60 \%)$ & $29 / 62(46 \%)$ & $6 / 11(55 \%)$ & 0.49 \\
Creatinine mg/dl & $0.99 \pm 0.25$ & $1.28 \pm 0.44$ & $1.27 \pm 0.38$ & 0.01 \\
NT-proBNP ng/l & $9762 \pm 8688$ & $5229 \pm 4818$ & $8246 \pm 12,646$ & 0.06 \\
IVS mm & $16.4 \pm 1.5$ & $17.3 \pm 2.5$ & $15.82 \pm 2.6$ & 0.18 \\
PW mm & $14.7 \pm 1.9$ & $16.3 \pm 2.6$ & $12.8 \pm 1.6^{*}$ & 0.001 \\
LVEDV ml & $73.7 \pm 28.8$ & $89.5 \pm 26.9$ & $99.2 \pm 21.7$ & 0.09 \\
LVESV ml & $36.2 \pm 15.1$ & $44.8 \pm 22.1$ & $41.3 \pm 18.1$ & 0.33 \\
EF $\%$ & $54.5 \pm 11.5$ & $50.9 \pm 12.1$ & $57.6 \pm 8.4$ & 0.40 \\
E cm/s & $79.0 \pm 23.0$ & $82.6 \pm 15.5$ & $88.2 \pm 19.2$ & 0.56 \\
A cm/s & $59.3 \pm 25.1$ & $50.1 \pm 30.4$ & $59.1 \pm 26.7$ & 0.43 \\
E/A & $1.7 \pm 1.0$ & $2.1 \pm 0.97$ & $1.69 \pm 0.8$ & 0.15 \\
DT ms & $171 \pm 65$ & $166 \pm 41$ & $197 \pm 88$ & 0.30 \\
E/E' & $16.8 \pm 6,1$ & $17.7 \pm 5.7$ & $14.2 \pm 6.2$ & 0.17 \\
PASP mm Hg & $37.2 \pm 10.9$ & $36.2 \pm 10.1$ & $39.7 \pm 13.4$ & 0.71 \\
TAPSE mm & $16.1 \pm 3.6$ & $16.6 \pm 3.8$ & $19.1 \pm 3.2$ & 0.12 \\
\hline
\end{tabular}

* Post hoc analysis $\mathrm{p}<0.05$ TTR vs LVH.
Our study for the first time demonstrated that in patients with TTR amyloidosis, 99mTc-HMDP scintigraphy shows some grade of lung retention in almost $60 \%$ of subjects. Moreover the incidence of lung uptake seems to be significantly correlated to the grade of heart retention according to Perugini visual score (Fig. 2). These data are in accordance with the research of Hutt and co-authors which recently demonstrated in a cohort of TTR amyloidosis patients, that soft tissue to femur ratio was significantly different between each Perugini grade of 99mTc-DPD scan, progressively increasing from grade 1 to grade 3 Perugini visual score [23]. These data could therefore suggest that both soft tissue tracer

Table 2

Scintigraphic and radiologic characteristics of the studied population.

\begin{tabular}{lllll}
\hline & $\begin{array}{l}\text { AL } \\
(\mathrm{n}=20)\end{array}$ & $\begin{array}{l}\text { TTR } \\
(\mathrm{N}=62)\end{array}$ & $\begin{array}{l}\text { LVH } \\
(\mathrm{N}=11)\end{array}$ & $\mathrm{p}=$ \\
\hline $\begin{array}{l}\text { Perugini visual score } \\
\text { Score 0 }\end{array}$ & 19 & 0 & 11 & 0.0001 \\
$\quad$ Score 1 & 1 & 2 & 0 & \\
Score 2 & 0 & 24 & 0 & \\
$\quad$ Score 3 & 0 & 36 & 0 & 0.0001 \\
Pulmonary uptake visual score & & & & \\
Score 0 & 19 & 26 & 11 & \\
$\quad$ Score 1 & 1 & 29 & 0 & \\
$\quad$ Score 2 & 0 & 7 & 0 & \\
Pulmonary radiologic score (49pts) & & & & \\
$\quad$ Score 0 & 1 & 14 & 3 & \\
$\quad$ Score 1 & 3 & 19 & - & \\
Score 2 & 2 & 7 & - & \\
\hline
\end{tabular}




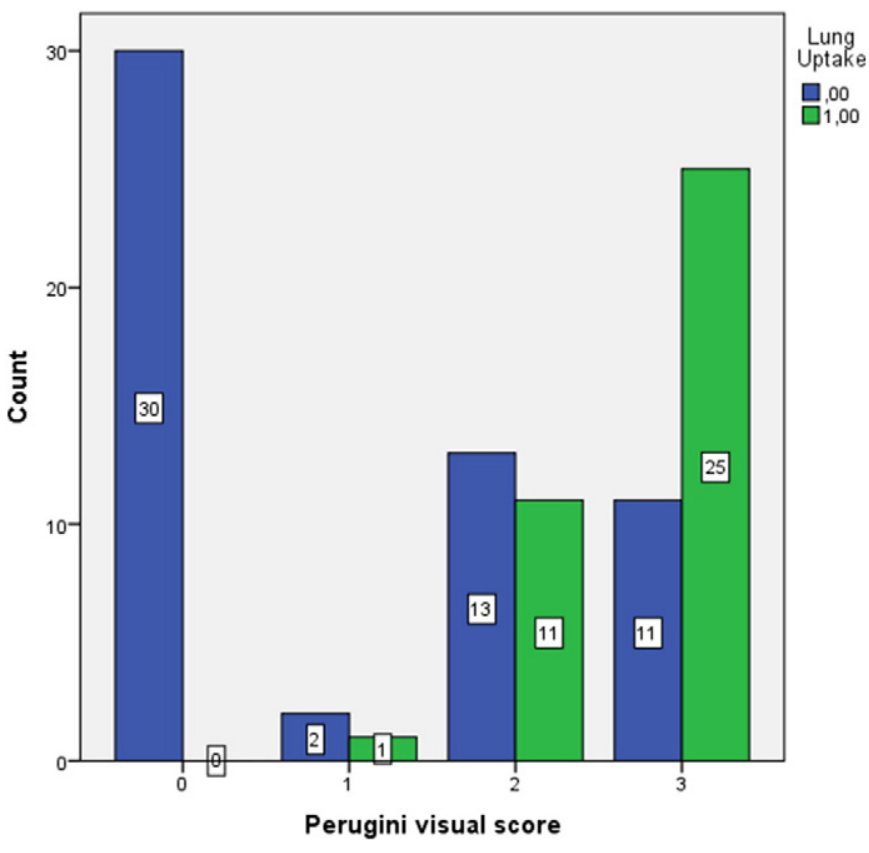

Fig. 2.99mTc-HMDP lung uptake distribution according to heart uptake distribution using Perugini visual score, in the whole population $(\mathrm{p}<0.001)$.

retention and lung retention are related to the amount of heart retention evaluated according to Perugini visual score.

The significant difference of distribution of 99mTc-HMPD lung uptake in AL and TTR amyloidosis suggests an aetiological tropism, comparable to the already well documented TTR related cardiac tropism. In fact lung retention was evidenced only in one out of twenty patients with AL amyloidosis, the only AL patient in our cohort, that also demonstrated a simultaneous mild but clearly evident heart retention. Interestingly no lung or heart retention was evidenced in the subgroup of patients with $\mathrm{LVH}$ not amenable to cardiac amyloidosis.

The mechanism underling lung bone tracer uptake is not well elucidated.

As previously suggested by Bach-Gansmo et al. [17] bone tracer lung retention could reflect blood-pool activity or relatively high local concentration in pulmonary tissue as part of soft tissue uptake in systemic amyloidosis.

In our opinion the first hypothesis is far less convincing: if lung retention was related to increased lung blood pool due to cardiac insufficiency, it should be similarly present in AL and LVH groups. In fact our three groups presented comparable LV systolic and diastolic function, RV longitudinal function and pulmonary systolic pressure. Moreover NT-proBNP despite not significant, tends to be lower in patients with TTR amyloidosis, the subgroup with the higher percentage of lung 99mTc-HMDP uptake.

Therefore 99mTc-HMDP pulmonary uptake could be more reasonably related to the presence of amyloid deposits in the lung. The increased incidence of lung retention in TTR subjects could be due to the highest tropism of 99mTc-HMDP for TTR amyloid deposit, a behaviour already well documented for bone tracer heart retention [19].

Unfortunately the lack of lung biopsy or post mortem lung evaluation let us only speculate on this hypothesis that should be confirmed by further studies.

Irrespectively to the underlying mechanism, the presence of bone tracer lung retention could lead to some technical concern. In fact a diffuse and easy to perform quantitative method to assess bone trace heart retention is based on the uptake ratio between cardiac region and a specular region in the contralateral lung (H/CL ratio) $[6,9,24]$. The presence of lung uptake could drastically influence this quantitative method, reducing the ratio between heart and lung. In our opinion if lung retention is evidenced, other quantitative methods such as heart and whole body retention (H/WBR) ratio or heart to skull ratio $(\mathrm{H} / \mathrm{S})$ [9] should be preferred.

\section{Conclusions}

The present findings demonstrated that in patients with TTR amyloidosis, 99mTc-HMDP scintigraphy shows lung uptake in almost $60 \%$ of subjects. Lung uptake is significantly less frequent in AL amyloidosis patients, and completely absent in patients with LVH but without CA. Moreover the incidence of lung uptake seems to be significantly correlated to the grade of heart retention according to Perugini visual score. We can speculate that the increase incidence of lung retention in TTR subjects could be explained by the highest tropism of 99mTc-HMDP for TTR amyloid deposit, a behaviour already well documented for bone tracer heart retention.

\section{Conflict of interest}

None declared.

\section{References}

[1] F. Perfetto, F. Cappelli, F. Bergesio, G. Ciuti, M.C. Porciani, L. Padeletti, et al., Cardiac amyloidosis: the heart of the matter, Intern. Emerg. Med. 8 (3) (2013 Apr) 191-203.

[2] E. Perugini, P.L. Guidalotti, F. Salvi, R.M. Cooke, C. Pettinato, L. Riva, et al., Noninvasive etiologic diagnosis of cardiac amyloidosis using 99mTc-3,3-diphosphono-1,2propanodicarboxylic acid scintigraphy, J. Am. Coll. Cardiol. 46 (6) (2005 Sep 20) 1076-1084.

[3] C. Rapezzi, C.C. Quarta, P.L. Guidalotti, C. Pettinato, S. Fanti, O. Leone, et al., Role of $(99 \mathrm{~m}) \mathrm{Tc}$-DPD scintigraphy in diagnosis and prognosis of hereditary transthyretin related cardiac amyloidosis, JACC Cardiovasc Imaging. 4 (2011) 659-670.

[4] S. Longhi, P.L. Guidalotti, C.C. Quarta, C. Gagliardi, A. Milandri, M. Lorenzini, L. Potena, et al., Identification of TTR related subclinical amyloidosis with $99 \mathrm{mTc}$-DPD scintigraphy, JACC Cardiovasc Imaging. 7 (2014) 531-532.

[5] D.F. Hutt, A.M. Quigley, J. Page, M.L. Hall, M. Burniston, D. Gopaul, et al., Utility and limitations of 3,3-diphosphono-1,2-propanodicarboxylic acid scintigraphy in systemic amyloidosis, Eur Heart J Cardiovasc Imaging. 15 (11) (2014 Nov) 1289-1298.

[6] S. Bokhari, A. Castano, T. Pozniakoff, S. Deslisle, F. Latif, M.S. Maurer, (99m)Tc-pyrophosphate scintigraphy for differentiating light-chain cardiac amyloidosis from the transthyretin-related familial and senile cardiac amyloidoses, Circ Cardiovasc Imaging. 6 (2013) 195-201.

[7] M. Hongo, J. Hirayama, T. Fujii, O. Kinoshita, M. Tanaka, S. Okubo, Early identification of amyloid heart disease by technetium-99m-pyrophosphate scintigraphy: a study with familial amyloid polyneuropathy, Am. Heart J. 113 (1987) 654-662.

[8] T.A. Wizenberg, J. Muz, Y.H. Sohn, W. Samlowski, A.M. Weissler, Value of positive myocardial technetium-99m-pyrophosphate scintigraphy in the noninvasive diagnosis of cardiac amyloidosis, Am. Heart J. 103 (1982) 468-473.

[9] A.W. Glaudemans, R.W. van Rheenen, M.P. van den Berg, W. Noordzij, M. Koole, H. Blokzijl, et al., Bone scintigraphy with $(99 \mathrm{~m})$ technetium-hydroxymethylene diphosphonate allows early diagnosis of cardiac involvement in patients with transthyretin-derived systemic amyloidosis, Amyloid 21 (2014) 35-44.

[10] A. Galat, J. Rosso, A. Guellich, A. Van Der Gucht, S. Rappeneau, D. Bodez, S. Guendouz, et al., Usefulness of (99m)Tc-HMDP scintigraphy for the etiologic diagnosis and prognosis of cardiac amyloidosis, Amyloid 22 (4) (2015) 210-220.

[11] A. Khoor, T. Colby, Amyloidosis of the lung, Arch Pathol Lab Med. 141 (2017) 247-254.

[12] H.A. Pickford, S.J. Swensen, J.P. Utz, Thoracic cross-sectional imaging of amyloidosis, AJR Am. J. Roentgenol. 168 (2) (1997 Feb) 351-355.

[13] A.C. Aylwin, P. Gishen, S.J. Copley, Imaging appearance of thoracic amyloidosis, J. Thorac. Imaging 20 (1) (2005 Feb) 41-46.

[14] R. Bauer, N. Dikow, A. Brauer, M. Kreuter, S. Buss, C. Evers, et al., The "Wagshurst study": p.Val40Ile transthyretin gene variant causes late-onset cardiomyopathy, Amyloid 21 (4) (2014 Dec) 267-275.

[15] Zwas ST, Shpilberg O, Huszar M, Rozenman J. Isolated ectopic lung uptake of technetium $99 \mathrm{~m}$ methylene diphosphonate on bone scintigraphy in primary amyloidosis. Eur J Nucl Med. 1990;17(5):282-5.

[16] M. Puille, K. Altland, R.P. Linke, M.K. Steen-Müller, R. Kiett, D. Steiner, et al., 99mTcDPD scintigraphy in transthyretin-related familial amyloidotic polyneuropathy, Eur. J. Nucl. Med. Mol. Imaging 29 (3) (2002 Mar) 376-379.

[17] T. Bach-Gansmo, T.N. Wien, A. Løndalen, E. Halvorsen, Myocardial uptake of bone scintigraphic agents associated with increased pulmonary uptake, Clin. Physiol. Funct. Imaging 36 (3) (2016 May) 237-241.

[18] S. Ohdama, S. Akagawa, O. Matsubara, Y. Yoshizawa, Primary diffuse alveolar septal amyloidosis with multiple cysts and calcification, Eur. Respir. J. 9 (1996) 1569-1571.

[19] J.D. Gillmore, M.S. Maurer, R.H. Falk, G. Merlini, T. Damy, A. Dispenzieri, et al., Nonbiopsy diagnosis of cardiac transthyretin amyloidosis, Circulation 133 (24) (2016 Jun 14) 2404-2412. 
[20] M. Gertz, G. Merlini, Definition of organ involvement and response to treatment in AL amyloidosis: an updated consensus opinion, Amyloid 17 (Suppl. 1) (2010) 48-49.

[21] R.M. Lang, L.P. Badano, V. Mor-Avi, J. Afilalo, A. Armstrong, L. Ernande, et al., Recommendations for cardiac chamber quantification by echocardiography in adults: an update from the American Society of Echocardiography and the European Association of Cardiovascular Imaging, J Am Soc Echocardiogr. 28 (1) (2015 Jan) (1-39.e14).

[22] S. Janssen, D.A. Piers, M.H. van Rijswijk, S. Meijer, E. Mandema, Soft-tissue uptake of $99 \mathrm{mTc}$-diphosphonate and 99mTc-pyrophosphate in amyloidosis, Eur. J. Nucl. Med. 16 (8-10) (1990) 663-670.
[23] D.F. Hutt, M. Fontana, M. Burniston, A.M. Quigley, A. Petrie, J.C. Ross, et al., Prognostic utility of the Perugini grading of 99mTc-DPD scintigraphy in transthyretin (ATTR) amyloidosis and its relationship with skeletal muscle and soft tissue amyloid, Eur Heart J Cardiovasc Imaging 18 (12) (2017 Dec 1) 1344-1350.

[24] M.N. Vranian, B.W. Sperry, M. Hanna, R. Hachamovitch, A. Ikram, R.C. Brunken, et al., Technetium pyrophosphate uptake in transthyretin cardiac amyloidosis: associations with echocardiographic disease severity and outcomes, J. Nucl. Cardiol. (2017 Jan 3)https://doi.org/10.1007/s12350-016-0768-9. 